\title{
Delamination of Large Area Layers of Hexagonal Boron Nitride Grown by MOVPE
}

\author{
J. IwAŃSKi*, A.K. DĄBrowska, M. TOKARCZYK, \\ J. Binder, R. StęPniewski And A. WysmoŁek \\ Faculty of Physics, University of Warsaw, L. Pasteura 5, 02-093 Warsaw, Poland \\ Doi: 10.12693/APhysPolA.139.457 \\ *e-mail: ji382362@okwf.fuw.edu.pl
}

\begin{abstract}
The possibility to fabricate van der Waals heterostructures on the wafer-scale would open up a wide range of potential applications. Hexagonal boron nitride $(\mathrm{hBN})$ is one of the most important components in many structures of this kind. Obtaining high quality $\mathrm{hBN}$ and the ability to transfer large areas to arbitrary surfaces is a must for future applications of many less durable and less stable van der Waals materials. In this work, we point out key factors that allow us to delaminate large areas of hBN layers grown by metal-organic vapor phase epitaxy on two-inch sapphire substrates. Using scanning electron microscopy and X-ray diffraction, we also compare hBN layers before and after the delamination process.
\end{abstract}

topics: delamination, boron nitride, MOVPE, large areas

\section{Introduction}

Boron nitride $(\mathrm{BN})$ is a wide bandgap material with high chemical stability and thermal conductivity. Particularly interesting is its layered form with $s p^{2}$ hybridization, also called hexagonal boron nitride (hBN). It has become an important element of van der Waals heterostructures, which are made of two-dimensional materials with diametrically different properties - $\mathrm{hBN}$ acts as an insulating barrier in them [1]. More complex van der Waals structures, also called NanoLego, open up a wide range of possibilities, for example for flexible electronics [2], ultrafast transistors [3, 4] or single photon sources used in quantum cryptography [5].

However, the application of hBN in everyday life requires the development of a method for obtaining high quality large area layers that are grown on commercially available substrates (e.g. sapphire) and mastering the process of delamination that allows transferring a hBN layer to target substrates or other two-dimensional materials.

One opportunity to achieve the above is to grow $s p^{2}$ BN layers by metal-organic vapor phase epitaxy (MOVPE). By growing samples under appropriate conditions one can obtain a material that can be easily delaminated from the sapphire substrate. As shown in Fig. 1a, after putting the sample on the surface of water, it penetrates between the hBN layer and the substrate peeling off material at the same time. This method of delamination was presented by Chugh et al. [6] but its mechanism is still unknown. As shown by Bera et al. [7], this kind of delamination also reduces strain within the material. In this work, we point out key parameters that are important for the proposed method.

\section{Methods}

\subsection{Sample growth}

All samples presented in this article were grown by MOVPE using an Aixtron CCS $3 \times 2$ " system. Two-inch diameter sapphire wafers were used as substrates, hydrogen as the carrier gas, triethyloboron (TEB) was the precursor of boron and ammonia - the precursor of nitrogen. Boron nitride was grown in different growth parameter regimes $[6,8-10]$.

The examined BN samples were epitaxial layers oriented parallel to the sapphire surface with thickness in the range from 1 to $30 \mathrm{~nm}$. Prior to the growth, the $\mathrm{Al}_{2} \mathrm{O}_{3}$ substrate surface was nitrided in $\mathrm{NH}_{3}$ gas flow. The nitridation time before the growth was from 0 to $3 \mathrm{~min}$ which resulted in different thickness and quality of AlN buffer layer.

\subsection{Delamination procedure}

All the samples in this work were delaminated according to the same procedure. Before delamination, samples were heated on a hot plate at $80^{\circ} \mathrm{C}$ for $10 \mathrm{~min}$. Then they were put onto the surface of a 1-2 $\mathrm{mm}$ thick liquid solution layer. It is important to adjust the surface of the sample parallel 

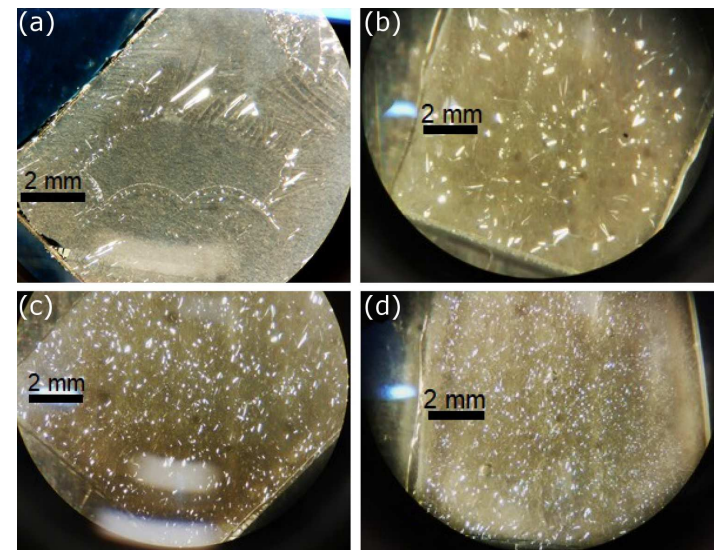

Fig. 1. Solvent penetrating between the BN layer and the sapphire substrate, peeling off the material. For the experiment (a) water, (b) isopropanol, (c) acetone, (d) toluene were used as a solvent.

to the surface of the liquid and prevent the sample from sinking. If this happens, the liquid penetrates from the edges of the sample between the hBN layer and the sapphire substrate (as shown in Fig. 1a) delaminating it immediately. When the process was too slow, an additional $1 \mathrm{~mm}$ thick layer of liquid was added and the Petri dish was moved to ruffle the surface of the liquid. After time, the hBN layer fully peeled off from the substrate, floating on the surface, whereas the substrate sank.

\section{Results and discussion}

\subsection{Delamination in different solvents}

We also studied how the usage of different solvents affects the delamination process. To this end, water was replaced by three types of solvents: polar aprotic (acetone; purity 99.5\%), polar protic (isopropanol; purity 99.7\%) and non-polar (toluene; purity $95 \%)$. We used two samples $\left(0.8 \times 8 \mathrm{~cm}^{2}\right)$ for each approach, both from the same wafer.

In Fig. 1, we present images taken with a camera connected to an optical microscope. One can see that they differ from each other depending on the solvent used. As shown in Fig. 1a, the sample interacts with water, which penetrates under the BN layer peeling it off. Water, while moving towards the middle of the sample, lifts up big areas of the material. This process can be very quick. The attempt with isopropanol (Fig. 1b) was similar to the one with water (Fig. 1a). Millimetersize areas of material were raised up. Even though $95 \%$ of the layer did not have contact with the substrate anymore, the layer did not detach. Acetone can easily penetrate between the $\mathrm{BN}$ layer and the $\mathrm{Al}_{2} \mathrm{O}_{3}$ substrate (Fig. 1c). However, it raises only a small amount of material. The layer resembled a crumpled foil bag. Moreover, the sample that was put on the acetone surface sank and the BN layer did not peel off. When the sample was placed onto the toluene surface, no interactions and no changes could be seen. After $30 \mathrm{~min}$, a high-density of small wrinkles could be observed as presented in Fig. 1d. Toluene penetration was the slowest among all the solvents tested and led to the least promising results.

Attempts with different solvents lead to the conclusion that water is one of the most important factors in the proposed method. This observation can be explained by the character of interaction between water and hBN presented by Tan et al. [11] who investigated peeling forces of hBN caused by water.

MOVPE-grown BN layers are usually very thin and easy to be damaged. By using pure water, the delamination process was rapid. To make it more controllable, we added isopropanol. An optimization of the isopropanol to water ratio allows to obtain a compromise between velocity of delamination and surface tension. As a consequence, the whole process becomes slower, easier to control and less prone to damage, which is important from the point of view of peeling off large area continuous layers. Our established isopropanol to water ratio that guarantees a good compromise is 1:8.

\subsection{Morphology before and after delamination in water/isopropanol solution}

The surface morphology of the samples was studied by scanning electron microscopy (SEM) using a FEI Helios NanoLab 600 system. An example of a typical morphology of the studied BN samples is shown in Fig. 2a. Characteristic wrinkles on the BN surface are visible regardless of the number of debris on the sample. The formation of wrinkles can be explained by the difference in thermal expansion coefficients of $\mathrm{BN}$ and $\mathrm{Al}_{2} \mathrm{O}_{3}[12,13]$. Figure $2 \mathrm{~b}$ shows the same sample but after the process of delamination. No wrinkles can be seen. By using the proposed delamination method we obtain a wrinklefree surface with less strain, in agreement with [7]. This fact constitutes a significant advantage over other methods for transferring large area BN layers that use, e.g., polymers like PMMA [14] or sputtered metals [15]. A strain-relaxed BN layer should also be more suitable for the growth of other 2D materials like transition metals dichalcogenides [16].
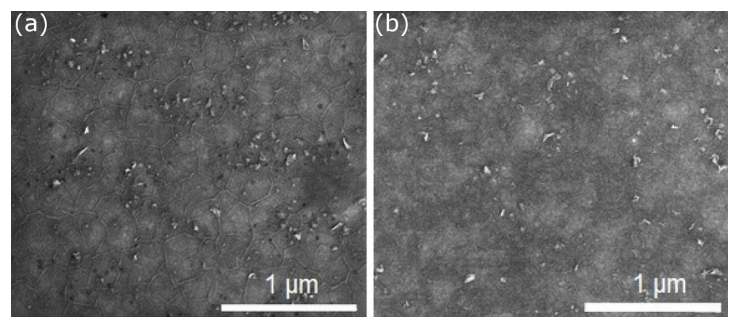

Fig. 2. SEM image of an BN layer (a) as grown, (b) redeposited on sapphire after the process of delamination. 


\subsection{X-ray diffraction measurements}

A Philips X'pert diffractometer equipped with a parallel beam Bragg X-ray mirror and $\mathrm{Cu}$ sealed $\mathrm{X}$-ray tube was used for structural characterization. The X-ray diffraction (XRD) peaks, as shown in Fig. 3a, are typical 2theta-omega scan results obtained from BN layers on sapphire substrates [17]. The BN sample delaminated and transferred to a silicon substrate (Fig. 3b) shows only a 002 peak from the BN layers. The angular position and FWHM of the BN peak remained almost unchanged (compared to the as-grown sample, Fig. 3a) which proves that the structure of the grown $\mathrm{BN}$ was preserved during the delamination process. The sapphire still showed a diffraction signal from the BN layer after delamination (Fig. 3c). It had the same FWHM but a decreased intensity. This means that the delamination had not taken place over the entire surface of the sample and a small amount of the BN layer remained, mainly in the vicinity of the edges of the sample. The intensity of the AlN peak slightly decreased, which may be related to a partial dissolution of the $\mathrm{AlN}$ in water.

3.4. Influence of growth conditions and BN properties on ability of delamination in water/isopropanol solution

We studied how different properties of BN samples can affect the ability to be delaminated with the proposed method (delaminatability). To make it more countable, we have introduced a five-point

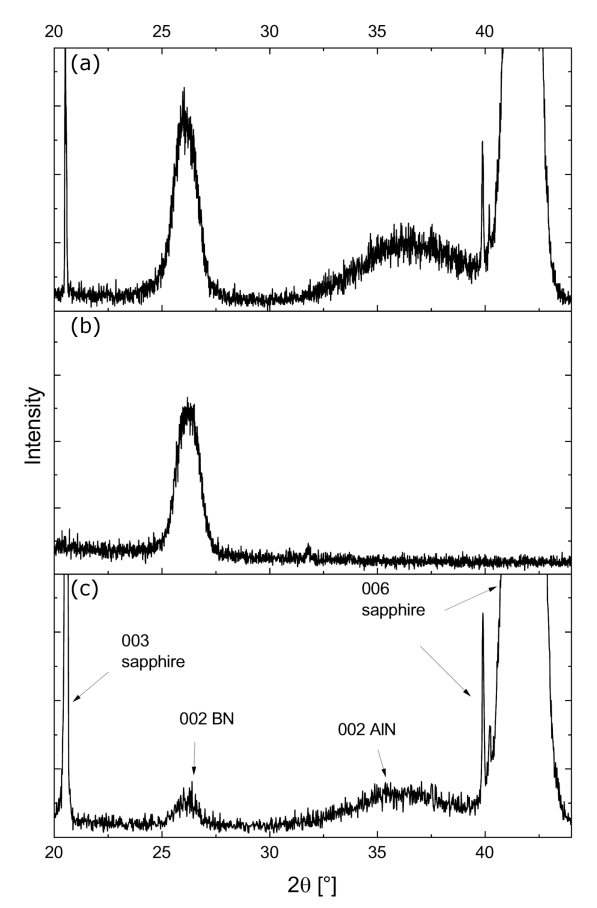

Fig. 3. X-ray diffraction 2theta/omega scans for: (a) BN on sapphire substrate (as grown), (b) delaminated BN transferred onto Si substrate, (c) sapphire substrate after delamination.
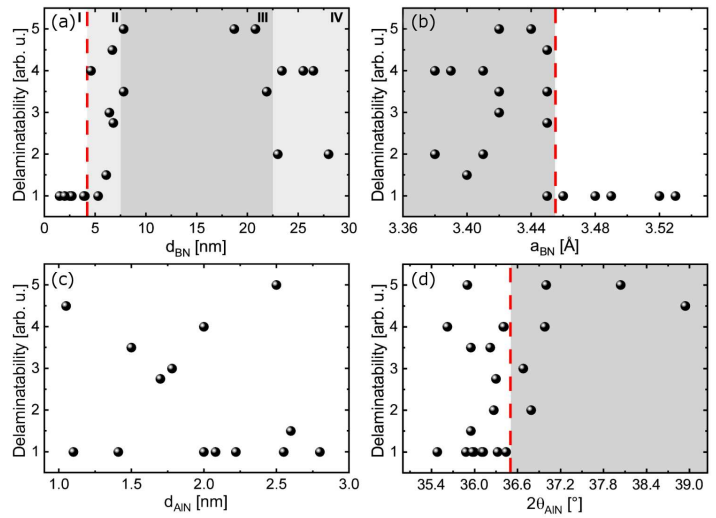

Fig. 4. Ability to be delaminated as a function of (a) BN layer thickness, (b) BN lattice constant, (c) unintentional AlN thickness, (d) AlN XRD peak position.

scale. Every conducted delamination process was different. They differ from each other in the duration or density of folds and their sizes. Therefore, only 1 and 5 on our scale are strictly defined. The situation in which the sample does not delaminate at all and no sign of delamination can be seen corresponds to a 1 on the scale. If it takes, however, only up to 5 seconds until the layer fully delaminates, the sample is ranked 5 . All the other points on the scale are set by taking into account the duration, density of folds, and their sizes.

There is no direct relationship between delaminatability and a single growth parameter, such as temperature, pressure, $\mathrm{NH}_{3}$ and TEB flows. As it was suggested by Chugh et al., delaminatability depends, among others, on the BN layer thickness $d_{\mathrm{BN}}$. Our study shows that it is indeed a critical parameter. As shown in Fig. 4a, there is a thickness range of about $8-22 \mathrm{~nm}$ that maximizes the probability of BN being delaminated (area III). One can see a different behavior in the delaminatability with thickness in the case of area II and area IV. That means that for delamination purposes it is better to grow films with thickness of tens of nanometers. It is connected with strain in the material. For very thin BN layers (area I), the energy related to compressive strain is insufficient to overcome the interaction between the layer and the substrate so the material does not wrinkle. As a consequence, the water cannot enter underneath the layer and delamination cannot proceed. On the other hand, thick layers are more rigid, preventing relaxation and, in consequence, delamination.

In Fig. 4b, one can also see that the better crystallographic structure of BN we have, the more likely is delamination. Higher values of $\mathrm{BN}$ lattice constant $a_{\mathrm{BN}}$ are due to turbostratic $\mathrm{BN}$ (t-BN) contribution to the material. The turbostratic phase is characterized by the torsion of atomic planes and their discontinuity. The resulting atomically thin flakes within the film cannot efficiently transfer the energy released during the relaxation of the material. 
Another key factor in the process of delamination is unintentional $\mathrm{AlN}$ formed on the $\mathrm{BN}-\mathrm{Al}_{2} \mathrm{O}_{3}$ interface during the growth process. When the sapphire substrate is exposed to ammonia at high temperatures and high pressure, the surface begins to etch. The oxygen atoms are discharged from the reactor and nitrogen atoms from $\mathrm{NH}_{3}$ replace them and bind to aluminum [18]. This process is possible in two cases: during the intentional nitridation before the BN growth and nonintentionally at the initial pulsed growth stage [10], when the BN layer is not yet securing the sapphire, and subsequent ammonia pulses can lead to AlN. The quality of the formed AlN is highly dependent on the compilation of many parameters - growth conditions, time of intentional nitridation, the time of NH3 pulses, etc. As Fukumoto et al. suggest, AlN powder can be dissolved in water [19]. If we treated the tiny and irregular layer of AlN as a powder, we should expect a dependence between delaminatability and AlN thickness $d_{\mathrm{AlN}}$. In fact, we find the delaminatability not to depend on AlN thickness as shown in Fig. 4c but it seems to be sensitive to its defects.

In Fig. 4d, one can see that moving away from the literature value for the AlN XRD peak position $2 \theta_{\text {AlN }}\left(36.04^{\circ}\right)[20]$ towards higher values, the probability for being delaminated increases. It strongly suggests that there is more space between atoms in more amorphous AlN which favors penetration of water between the BN and the substrate. However, it is also possible to obtain BN samples with a good AlN XRD peak position value that is able to delaminate (gray area).

This fact is a clear indication that other parameters like layer thickness or strain in the material are more significant.

\section{Conclusions}

We have studied large-scale $\left(\sim \mathrm{cm}^{2}\right)$ delamination of boron nitride grown on sapphire substrates by MOVPE. The use of different solvents revealed that the presented delamination technique proceeded fastest in pure water, however, better control over the process was achieved with a water/isopropanol solution, which supports peeling off a BN layer from the substrate. We show that $\mathrm{hBN}$ quality plays an important role for delamination and identify a thickness range of tens of nanometers to be favorable for delamination. We find evidence that the quality of unintentional AlN on the BNsapphire interface cannot be neglected either. The right amount of defects seems to increase the probability of delamination. All of the mentioned factors have a strong impact on large-area $\mathrm{hBN}$ delamination. XRD measurements show that the process of delamination happens at the $\mathrm{Al}_{2} \mathrm{O}_{3} / \mathrm{AlN}-\mathrm{BN}$ interface and SEM images prove that the delaminated layer is continuous and wrinkle-free.
It is clear that more studies are needed to investigate the conditions and growth parameters that allow us to obtain structures which would be easily delaminated or resistant against delamination. Our studies present a first step towards a prediction if a BN layer will be easy to delaminate from the substrate which is important for different applications.

\section{Acknowledgments}

This work has been partially supported by the National Science Centre under grant No. 2019/33/B/ST5/02766.

\section{References}

[1] J. Binder, F. Withers, M.R. Molas et al., Nano Lett. 17, 1425 (2017).

[2] G.-H. Lee, Y.-J. Yu, X. Cui, N. Petrone, C.-H. Lee, ACS Nano 7, 7931 (2013).

[3] G. Nazir, M.A. Rehman, M.F. Khan, G. Dastgeer, S. Afta, A.M. Afzal, Y. Seo, J. Eom, ACS Appl. Mater. Interfaces 10, 32501 (2018).

[4] Y. Sasama, K. Komatsu, S. Moriyama et al., Phys. Rev. Mater. 3, 121601(R) (2019).

[5] M. Koperski, M. Molas, A. Arora, K. Nogajewski, A. Slobodeniuk, F. Clément, M. Potemski, Nanophotonics 6, 1289 (2017).

[6] D. Chugh, J. Wong-Leung, L. Li, M. Lysevych, H.H. Tan, C. Jagadish, $2 D$ Mater. 5, 045018 (2018).

[7] K. Bera, D. Chugh, A. Patra, H.H. Tan, C. Jagadish, A. Roy, Solid State Commun. 310, 113847 (2020).

[8] K. Pakuła, A. Dąbrowska, M. Tokarczyk, R. Bożek, J. Binder, G. Kowalski, A. Wysmołek, R. Stępniewski, arXiv:1906.05319, 2019.

[9] A.K. Dąbrowska, M. Tokarczyk, G. Kowalski, J. Binder, R. Bożek, J. Borysiuk, R. Stępniewski, A. Wysmołek, 2D Mater. 8, 015017 (2021).

[10] Y. Kobayashi, T. Akasaka, T. Makimoto, J. Cryst. Growth 310, 5048 (2008).

[11] J. Tan, Y. Wang, Y. Guo, Nanotechnology 32, 025302 (2021).

[12] B. Yates, M.J. Overy, O. Pirgon, Philos. Mag. 32, 847 (1975).

[13] W.M. Vim, R.J. Paff, J. Appl. Phys. 45, 1456 (1974).

[14] T. Uwanno, Y. Hattori, T. Taniguchi, K. Watanabe, K. Nagashio, 2D Mater. 2, 041002 (2015).

[15] Jaewoo Shim, Sang-Hoon Bae, Wei Kong et al., Science 362, 665 (2018). 
[16] W. Pacuski, M. Grzeszczyk, K. Nogajewski et al., Nano Lett. 20, 3058 (2020).

[17] X. Yang, S. Nitta, M. Pristovsek, Y. Liu, Y. Liao, M. Kushimoto, Y. Honda, H. Amano, 2D Mater. 7, 015004 (2020).

[18] P. Vennéguès, B. Beaumont, Appl. Phys. Lett. 75, 4115 (1999).

[19] S. Fukumoto, T. Hookabe, H. Tsubakino, J. Mater. Sci. 35, 2743 (2000).

[20] Joint Committee on Powder Diffraction Standards (JCPDS), AlN pattern 25-1133. 